Research Paper

\title{
The Effect of Single Embryo Transfer on Perinatal Outcomes in Japan
}

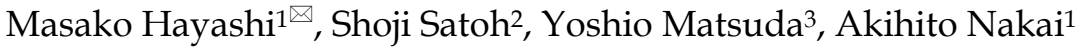 \\ 1. Department of Obstetrics and Gynecology, Nippon Medical School, Tokyo, Japan; \\ 2. Maternal and Perinatal Care Center, Oita Prefectural Hospital, Oita, Japan; \\ 3. Department of Obstetrics and Gynecology, International University of Health and Welfare, Tochigi, Japan.
}

$\square$ Corresponding author: Masako Hayashi Ph.D. Tama-Nagayama Hospital, Nippon Medical School, 1-7-1 Nagayama, Tama-City, Tokyo 206-8512, Japan. Tel: 81-(0) 42-371-21 11 Fax: 81-(0) 42-372-73 72 E-mail: hayashi@nms.ac.jp.

( ) Ivyspring International Publisher. This is an open-access article distributed under the terms of the Creative Commons License (http://creativecommons.org/ licenses/by-nc-nd/3.0/). Reproduction is permitted for personal, noncommercial use, provided that the article is in whole, unmodified, and properly cited.

Received: 2014.08.17; Accepted: 2014.11.04; Published: 2015.01.01

\begin{abstract}
Objective: In 2007 and 2008, the Japan Society for Reproductive Medicine and the Japan Society of Obstetrics and Gynecology issued a recommendation for single embryo transfer (SET). Thereafter, SET was implemented in $73 \%$ of in vitro fertilization (IVF) cases in Japan. The purpose of this study was to evaluate the effects of compliance with the SET recommendation on perinatal outcomes.

Methods: An electronic audit of the perinatal database of the Japanese Society of Obstetrics and Gynecology was conducted from 2001 through 2010. The database comprised data of 610,726 women. Totally, 20,923 women conceived through IVF. To compare perinatal outcomes, these women were categorized into two study groups depending on whether they conceived before (2004-2005, $n=3,865)$ or after $(2009-2010, n=6,842)$ the SET recommendation statement was issued.

Results: The proportion of women who conceived through IVF increased from $1.3 \%$ in 2001 to $4.8 \%$ in 2010 . Compliance with the SET recommendation led to a decrease in the incidence of twin pregnancies $(33.9 \%$ versus $13 \%, \mathrm{p}<0.01$ ), incidence of preterm delivery (odds ratio [OR]: 0.54 , 95\% confidence interval [Cl]: $0.50-0.59$ ), low birth weight (OR: $0.42,95 \% \mathrm{Cl}: 0.39-0.45$ ), and neonatal intensive care unit admission (OR $0.70,95 \% \mathrm{Cl} 0.65-0.76$ ), but an increase in the incidence of monochorionic twins $(1.6 \%$ versus $2.5 \%, \mathrm{p}<0.01)$.

Conclusion: Compliance with the SET recommendation improved perinatal outcomes by reducing the incidence of twin pregnancies.
\end{abstract}

Key words: in vitro fertilization, twin pregnancy, monochorionic twin, obstetric outcome, perinatal outcome.

\section{Introduction}

Since the birth of the first baby conceived through in vitro fertilization (IVF) in 1978, assisted reproductive technology (ART) has been a highly successful and widely employed modality for treating infertility. Initially, because inadequate embryo culture techniques resulted in a poor success rate following IVF, clinicians transferred multiple embryos to maximize the chances of conception. As advances in culture techniques improved the pregnancy rate of
IVF, multiple-gestation pregnancies increasingly became a problem $(1,2)$.

Recently, elective single embryo transfer (eSET) has been adopted by ART centers worldwide as a means to reduce the multiple pregnancy rates $(3,4)$. In European countries, national legislation restricts the number of embryos transferred $(3,5)$. In 1998, the American Society for Reproductive Medicine issued practice guidelines on the number of embryos to 
transfer, and has periodically revised these guidelines, most recently in 2013 (4). These guidelines recommend consideration of eSET in women with a favorable prognosis aged under 35. Single-embryo transfer (SET) has been slowly adopted in many countries, and a few studies have assessed the contribution of the eSET strategy on perinatal outcomes (6-8).

In Japan, the Japan Society for Reproductive Medicine (JSRM), concerned about the number of multiple births resulting from ART, prepared concrete recommendation regarding the number of embryos transferred in 2006, and developed specific guidelines about the number of embryos to be transferred in March 2007(9, 10). In 2008, the Japan Society of Obstetrics and Gynecology (JSOG) issued a recommendation for SET for all cases except in cases of repeated IVF failure or in patients $>35$ years where two embryos could be transferred (11).

This study evaluates the effect of compliance with the SET recommendation on perinatal outcomes in pregnancies conceived through IVF using a large Japanese database.

\section{Materials and Methods}

The study protocol was reviewed and approved by the Institutional Review Board of Nippon Medical School, Tokyo, Japan. We selected a case-control design to evaluate the effect of the SET recommendation statement on the perinatal outcomes of pregnancies conceived through IVF.

Data in this study derived from the perinatal database of JSOG. This nationwide registry was established in 1974. Detailed descriptions of the database have been published previously $(12,13)$. In brief, the attending physicians at 139 tertiary centers of the Perinatal Research Network in Japan collected data annually on all pregnant women in an offline clinical database with a common format. The data were sent to the perinatal committee of JSOG, where quality control for the database was assessed.

We used data of 638,268 deliveries reported between 2001 and 2010. Gestational age was determined on the basis of menstrual history, prenatal examination, and ultrasound findings, such as gestational sac diameter, crown-rump length, and biparietal diameter. Linked data included information on maternal characteristics, such as maternal age, parity, cigarette smoking, and alcohol consumption during pregnancy, history of treatment for infertility (ovulation stimulation, intrauterine insemination, or IVF-embryo transfer), medical complications, and pregnancy complications. Fetal and infant outcomes were also routinely recorded by attendants at delivery. These data conform to uniform coding specifications, have passed a rigorous quality check, and have been edited and reviewed. The current study limited the analysis to women who delivered a live birth or stillbirth at 22 weeks of gestation or more. After excluding missing data, 610,719 pregnancies were included in this study.

Two study groups were created according to whether they conceived before or after the SET recommendation statement was issued. We did not adopt the data from 2006 through 2008 to exclude influence of the shift period, because the guidelines on JSRM were announced for one year before 2007 by their journal and website, and JSOG issued a statement of recommendation for SET in 2008. The IVF group included both women who received eggs spontaneously fertilized by sperm in vitro, or eggs fertilized by intracytoplasmic sperm injection (ICSI), followed by transfer of the embryo back into the uterine cavity. However, further information on the use of ICSI was unavailable in our database.

Data were analyzed using SPSS Statistics version 17.0 (SPSS Inc., Chicago, IL, USA) and Excel (Microsoft, Redmond, WA, USA). Statistical analysis was performed using the Mann-Whitney $U$ test for continuous variables, such as maternal age, body mass index (BMI), birth weight, and the chi-squared test for categorical variables. A $p$ value of $<0.05$ was considered statistically significant. Logistic regression analysis was used to compare adjusted odds ratios (OR) and $95 \%$ confidence intervals (CI) for adverse obstetric and perinatal outcomes before and after the issue of the SET recommendation.

\section{Results}

In total, 20,923 (3.4\%) women conceived through IVF during the study period. The proportion of women who conceived through IVF increased dramatically from $1.3 \%$ in 2001 to $4.8 \%$ in 2010 (Figure 1). From 2001-2006, the incidence of twin pregnancies conceived through IVF increased concurrently with the proportion of women who conceived through IVF. After the issue of the SET recommendation statement in 2007 and 2008, although a marked increase in the proportion of pregnancies conceived through IVF was evident, the incidence of twin pregnancies conceived through IVF significantly decreased (Figure 1). The incidence of twin pregnancies among the pregnancies conceived with IVF was significantly decreased from $33 \%$ to $13 \%$ due to a reduction of dichorionic twins. In contrast, the incidence of monochorionic twins conceived through IVF continuously increased between 2001 and 2010, from 1.1\% to 2.6\% (Figure 2). 


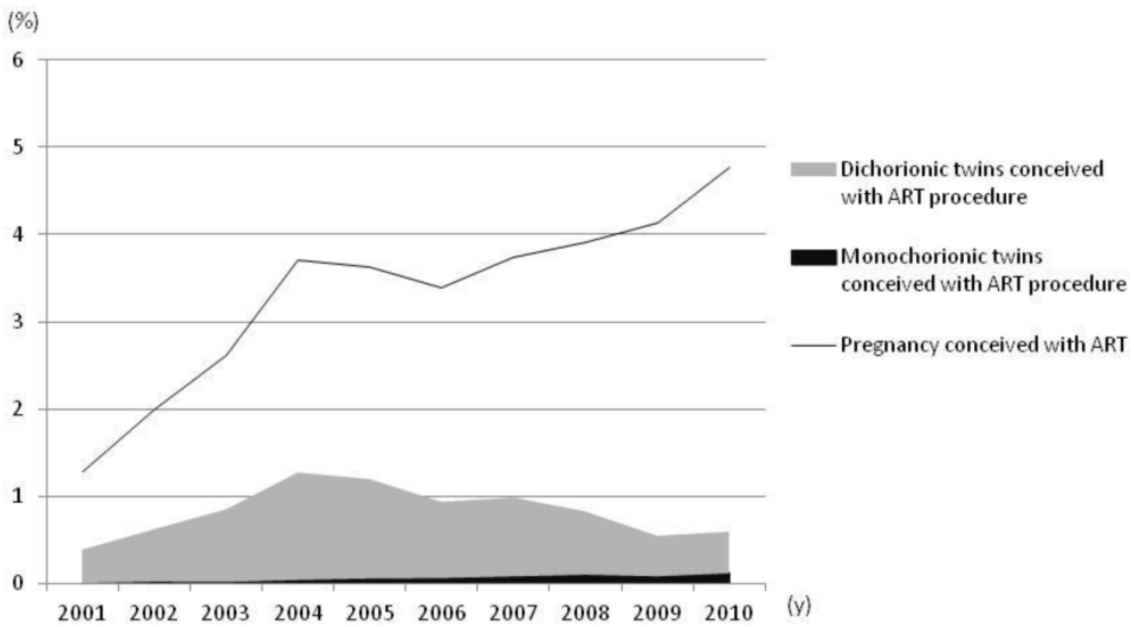

Figure 1. Changes in incidence of the pregnancies conceived with IVF and twin pregnancies after IVF treatment among the total pregnancy. Pregnancies from IVF increased dramatically from $1.3 \%$ in 2001 to $4.8 \%$ in 2010. Twin pregnancies conceived through IVF increased concurrently with the proportion of women who conceived through IVF, but after the SET recommendation statement was issued in 2007 and 2008, the incidence of twin pregnancies conceived through IVF significantly decreased.

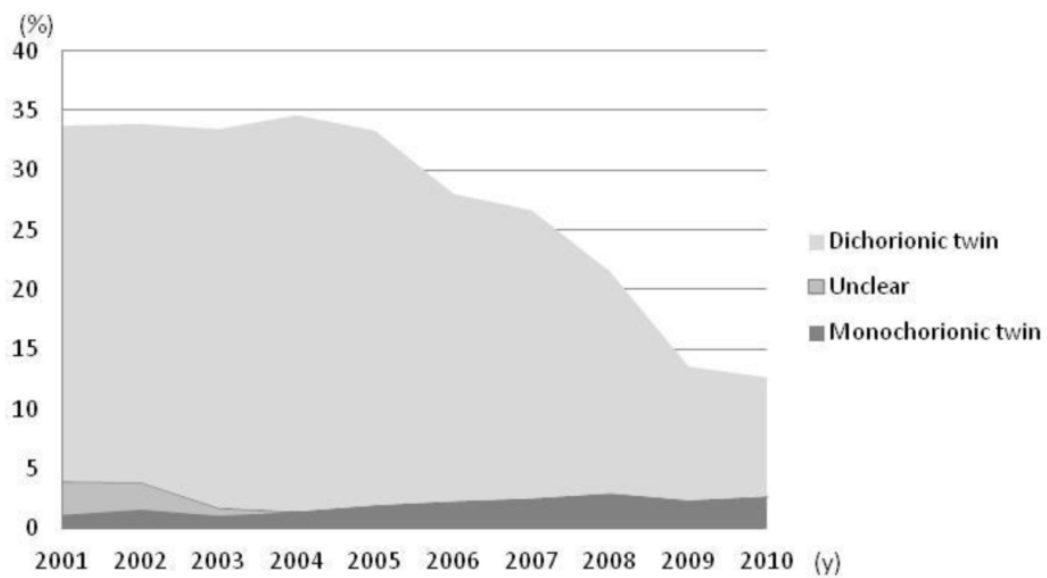

Figure 2. Changes in incidence of twin pregnancies conceived with IVF. The incidence of twin pregnancies among the pregnancies conceived with IVF was significantly decreased from $33 \%$ to $13 \%$ with the reduction of dichorionic twins. In contrast, the incidence of monochorionic twins conceived through IVF continuously increased between 2001 and 2010 , from $1.1 \%$ to $2.6 \%$.

To evaluate the effect of compliance with the SET recommendation on the perinatal outcomes of pregnancies conceived through IVF, women who conceived through IVF were categorized into two study groups depending on whether they conceived before (2004-2005, $n=3,865)$ or after (2009-2010, $n=6,842)$ the SET recommendation statement issued. The maternal characteristics of the two study groups are summarized in Table 1. Maternal age and the proportion of women with preexisting medical complications tended to be higher in 2009-2010 than in 2004-2005. The proportion of nulliparous women decreased in 2009-2010. No significant differences were observed in BMI and alcohol consumption between the two groups.

The overall incidences of obstetric and perinatal complications in the study population are shown in Table 2. The incidence of multiple pregnancies dras- tically declined after the issue of the SET recommendation (twins: $33 \%$ versus $13 \%$; triplets: $2.1 \%$ versus $0.3 \% ; \mathrm{p}<0.01)$. However, the incidence of monochorionic twins was significantly higher in 2009-2010 (2.5\%) than in 2004-2005 (1.6\%; OR 1.54, 95\% CI 1.15-2.07). The incidence of cesarean section decreased after the SET recommendation was issued (OR 0.71, 95\% CI 0.66-0.77). Marked decreases in the incidences of threatened miscarriage (OR $0.52,95 \% \mathrm{CI}$ 0.44-0.62), preterm labor (OR 0.51, 95\% CI 0.47-0.56), and preterm delivery at $<37$ weeks (OR $0.54,95 \%$ CI $0.50-0.59$ ) and at <34 weeks (OR 0.61, 95\% CI 0.54-0.69) were also evident in 2009-2010.

Delivery of low-birth weight infants $(<2,500 \mathrm{~g})$ was common among women who conceived through IVF. In 2004-2005, over a half of newborns were low-birth weight infants. Compared with the 2004-2005 group, the incidences of low-, very low-, 
and extremely low-birth weight infants significantly decreased in $2009-2010$, at $52.3 \%$ versus $31.5 \%, 9.4 \%$ versus $6.5 \%$, and $3.9 \%$ versus $2.9 \%$, respectively. The incidence of small-for-gestational-age infants also decreased in 2009-2010 (OR 0.55, 95\% CI 0.48-0.63). Compliance with the SET recommendation was associated with a decrease in adverse neonatal outcomes, such as neonatal intensive care unit (NICU) admission (OR 0.70, 95\% CI 0.65-0.76), and infant death (OR 0.71, 95\% CI 0.55-0.93).

Table 1. Maternal characteristics of the study population.

\begin{tabular}{|c|c|c|c|}
\hline & $2004-2005$ & $2009-2010$ & \\
\hline & $\mathrm{n}=3865$ & $\mathrm{n}=6842$ & $\mathrm{P}$ \\
\hline Maternal age $(y)^{*}$ & $35(19$ - 54) & $37(21-58)$ & $<0.01$ \\
\hline-34 & $1743(45.3)$ & $2075(30.3)$ & $<0.01$ \\
\hline $35-39$ & $1566(40.7)$ & 3234 (47.3) & \\
\hline 40- & $536(13.9)$ & $1531(22.4)$ & \\
\hline unclear / missed & 20 & 2 & \\
\hline \multicolumn{4}{|l|}{ Parity } \\
\hline Nulliparous & 3201 (82.9) & 5228 (76.5) & $<0.01$ \\
\hline Multiparous & 662 (17.1) & 1607 (23.5) & \\
\hline Unclear / missed & 2 & 7 & \\
\hline Body mass index* & $20.5(14.6-40.7)$ & $\begin{array}{l}20.5 \\
50.2)\end{array}$ & 0.39 \\
\hline-18.4 & $228(15.5)$ & $933(16.0)$ & 0.88 \\
\hline $18.5-24.9$ & $1096(74.5)$ & $4273(73.4)$ & \\
\hline $25.0-29.9$ & $113(7.7)$ & $472(8.1)$ & \\
\hline 30.0- & $35(2.4)$ & $140(2.4)$ & \\
\hline unclear / missed & 2393 & 1024 & \\
\hline \multicolumn{4}{|l|}{ Smoking in pregnancy } \\
\hline Yes & $79(2.3)$ & $64(1.0)$ & $<0.01$ \\
\hline No & 3356 (97.7) & $6515(99.0)$ & \\
\hline unclear/missed & 430 & 263 & \\
\hline \multicolumn{4}{|l|}{$\begin{array}{l}\text { Alcohol consumption in preg- } \\
\text { nancy }\end{array}$} \\
\hline Yes & $84(2.5)$ & $165(2.5)$ & 0.89 \\
\hline No & $3293(97.5)$ & 6351 (97.5) & \\
\hline unclear/missed & 488 & 326 & \\
\hline \multicolumn{4}{|l|}{$\begin{array}{l}\text { Preexisting medical complica- } \\
\text { tions }\end{array}$} \\
\hline Yes & 1209 (31.3) & $2749(40.2)$ & $<0.01$ \\
\hline No & $2656(68.7)$ & $4093(59.8)$ & \\
\hline \multicolumn{4}{|l|}{ Diabetes mellitus } \\
\hline Yes & $89(2.3)$ & 219 (3.2) & $<0.01$ \\
\hline No & 3775 (97.7) & $6623(96.8)$ & \\
\hline \multicolumn{4}{|l|}{ Hypertension } \\
\hline Yes & $21(0.5)$ & $80(1.2)$ & $<0.01$ \\
\hline No & 3844 (99.5) & $6762(98.8)$ & \\
\hline \multicolumn{4}{|l|}{ Thyroid disease } \\
\hline Yes & $80(2.1)$ & $307(4.5)$ & $<0.01$ \\
\hline No & 3785 (97.9) & 6535 (95.5) & \\
\hline
\end{tabular}

Values are $\% .{ }^{*}$ median (range). NA: not applicable.

\section{Discussion}

Our data show that compliance with the SET recommendation successfully reduced multiple pregnancies conceived through IVF and dramatically reduced multiple pregnancy-related complications.

The proportion of pregnancies conceived through IVF has rapidly increased in Japan. Until 2007, the incidence of twin pregnancies conceived through IVF increased pari passu with the rate of pregnancies conceived through IVF (Figure 1). Previous studies $(14,15)$ have suggested that multiple pregnancies are the most serious complication of IVF for both mother and child. The main risk factor of ART treatment for dizygotic twinning and high-order multiple pregnancies is the transfer of more than one embryo $(16,17)$. It is established that multiple gestations carry a high risk of perinatal morbidity and mortality and are associated with increased risk of miscarriage, preterm delivery, and cesarean section compared with singleton pregnancies $(14,18,19)$.

SET has been gradually accepted worldwide as a means to reduce multiple pregnancy rates (5). Recently, several studies $(6,20-24)$ have reported the efficacy of SET, which yields an excellent pregnancy rate and drastically decreases twin pregnancies. Other retrospective studies $(25,26)$ have demonstrated that SET after transfer of thawed embryos results in similar pregnancy rates as double-embryo transfer and significantly decreased multiple pregnancy rates. However, the number of SETs performed remains modest in many countries. In the US, only $13.5 \%$ of ART cycles employed SET in 2009 (27) and even in Europe, only $22 \%$ of transfers were SET in 2008 (3). Conversely, in Japan, the proportion of SET among IVF cycles was high: $50 \%$ in $2007,63.6 \%$ in $2008,70.6 \%$ in 2009, and $73 \%$ in 2010(28) although there was no contract punitive clause for non-observance.

Our results indicate that the incidence of multiple pregnancies drastically declined after the SET recommendation statement was issued. Simultaneous to the reduction in multiple pregnancies, reductions in the incidences of neonatal resuscitation, neonatal NICU admission, preterm delivery, and cesarean section were evident. These results suggest that a higher ratio of SET among IVF cycles in other countries, as Japan, could have profound public health implications by reducing the occurrence of iatrogenic twin pregnancies.

On the other hand, despite the reduction in twin pregnancies, we found that the incidence of monochorionic twins conceived through IVF increased from $1.6 \%$ in $2004-2005$ to $2.5 \%$ in $2009-2010$. To date, several possible explanations have been proposed for this increase in monochorionic twinning. Several authors have speculated that extended culture and/or an increase in the use of ICSI to improve the pregnancy rate could contribute to the increased incidence of monozygotic twins (29-32). Some studies $(29,30)$ have concluded that the risk of monozygotic twinning is increased when culture is extended to the blastocyst stage. Another report (31) suggested that the risk of monozygotic twinning may relate to the composition of culture medium. A further report (32) found no 
association between the type of culture medium and the risk of monozygotic twinning, although there was a 24-fold increased risk of monozygotic twinning in cycles involving both ICSI and extended culture. We acknowledge that our results cannot support any of these possibilities: the inability to identify fresh, frozen-thawed, cleavage-stage embryo transfer, blastocyst transfer, or ICSI cycles is a limitation of our database.

In conclusion, this study indicates that compliance with the SET recommendation improved peri- natal outcomes through a reduction in multiple gestation rates. These results suggest than a higher proportion of SET among IVF cycles in other countries as in Japan, could have profound public health implications by reducing the occurrence of iatrogenic twin pregnancies.

Our results also demonstrate that the incidence of monochorionic twins increased with the increased compliance with the SET recommendation, which requires further study.

Table 2. Comparison of perinatal outcome before $(2004-2005, n=3865)$ and after $(2009-2010, n=6842)$ the SET recommendation statement issued.

\begin{tabular}{|c|c|c|c|c|c|}
\hline & & 2004 - 2005 year & 2009 - 2010 year & & \\
\hline & Mother & $\mathrm{n}=3865$ & $\mathrm{n}=6842$ & & \\
\hline Outcome & Infant & $\mathrm{n}=5340$ & $\mathrm{n}=7780$ & & OR $(95 \% \mathrm{CI})$ \\
\hline \multicolumn{6}{|l|}{ Pregnancies } \\
\hline Singleton & & $2472(64.0)$ & 5927 (86.6) & $<0.01$ & $3.65(3.32-4.02)$ \\
\hline Twin & & $1311(33.9)$ & $892(13.0)$ & $<0.01$ & $0.29(0.27-0.32)$ \\
\hline Monochorionic twin & & $62(1.6)$ & $168(2.5)$ & $<0.01$ & $1.54(1.15-2.07)$ \\
\hline Dichorionic twin & & $1246(32.2)$ & $719(10.5)$ & $<0.01$ & $0.25(0.22-0.27)$ \\
\hline Unclear & & 3 & 5 & & \\
\hline Triplet & & $82(2.1)$ & $23(0.3)$ & $<0.01$ & $0.16(0.10-0.25)$ \\
\hline \multicolumn{6}{|l|}{ Type of delivery } \\
\hline Spontaneous cephalic & & $1338(34.6)$ & $2798(40.9)$ & $<0.01$ & $1.31(1.20-1.42)$ \\
\hline Instrumental & & $285(7.4)$ & $634(9.3)$ & $<0.01$ & $1.28(1.11-1.48)$ \\
\hline Cesarean & & $2220(57.4)$ & $3345(48.9)$ & $<0.01$ & $0.71(0.66-0.77)$ \\
\hline Others & & $22(0.6)$ & $65(1.0)$ & 0.04 & $1.68(1.03-2.72)$ \\
\hline Threatened miscarriage & & $281(7.3)$ & $269(3.9)$ & $<0.01$ & $0.52(0.44-0.62)$ \\
\hline Preterm labor & & $1269(33.0)$ & $1374(20.1)$ & $<0.01$ & $0.51(0.47-0.56)$ \\
\hline $\mathrm{PIH}$ & & $212(5.5)$ & $384(5.6)$ & 0.78 & $1.02(0.86-1.22)$ \\
\hline Placenta previa & & $119(3.1)$ & $243(3.6)$ & 0.2 & $1.16(0.93-1.45)$ \\
\hline Placenta accreta & & $29(0.8)$ & $95(1.4)$ & $<0.01$ & $1.86(1.23-2.83)$ \\
\hline Postpartum hemorrhage & & $207(5.4)$ & $443(6.5)$ & 0.02 & $1.22(1.03-1.45)$ \\
\hline Preterm delivery $<37 w$ & & $1271(32.9)$ & $1436(21.0)$ & $<0.01$ & $0.54(0.50-0.59)$ \\
\hline Preterm delivery $<34 \mathrm{w}$ & & $483(12.5)$ & $547(8.0)$ & $<0.01$ & $0.61(0.54-0.69)$ \\
\hline blood loss during delivery $\left(\mathrm{g}^{*}\right)$ & & $747(2-5448)$ & $716(7-6000)$ & 0.03 & \\
\hline Maternal death & & $0(0.0)$ & $2(0.0)$ & 0.54 & NA \\
\hline \multicolumn{6}{|l|}{ Infants } \\
\hline Birthweight $\left(\mathrm{g}^{*}\right)$ & & $2459(252-4910)$ & $2800(50-4790)$ & $<0.01$ & \\
\hline$<2500 \mathrm{~g}$ & & $2783(52.3)$ & $2440(31.5)$ & $<0.01$ & $0.42(0.39-0.45)$ \\
\hline$<1500 \mathrm{~g}$ & & $502(9.4)$ & $504(6.5)$ & $<0.01$ & $0.67(0.59-0.76)$ \\
\hline$<1000 \mathrm{~g}$ & & $205(3.9)$ & $221(2.9)$ & $<0.01$ & $0.73(0.60-0.89)$ \\
\hline SGA & & $534(10.0)$ & $447(5.7)$ & $<0.01$ & $0.55(0.48-0.63)$ \\
\hline $\mathrm{UmApH}<7.0$ & & $13(0.5)$ & $40(0.7)$ & 0.34 & $1.36(0.72-2.54)$ \\
\hline Apgar score $(5 \mathrm{~min})<7$ & & $229(4.3)$ & $278(3.6)$ & 0.04 & $0.83(0.69-0.99)$ \\
\hline Neonatal resuscitation & & $2071(39.3)$ & $2205(28.6)$ & $<0.01$ & $0.62(0.58-0.67)$ \\
\hline NICU admission & & $1460(27.3)$ & $1628(20.9)$ & $<0.01$ & $0.70(0.65-0.76)$ \\
\hline Infant death & & $112(2.1)$ & $117(1.5)$ & 0.01 & $0.71(0.55-0.93)$ \\
\hline
\end{tabular}

Values are n (\%). * median (range). NA: not applicable.

\section{Acknowledgments}

We sincerely thank Mr. Norio Sugimoto for his help to our statistical analysis. We also thank Professor Yukihiro Terada for valuable advice with regard to interpretation of the results.

\section{Contributions}

1) Conception and design: M.H., A.N.,
2) Acquisition of data: S.S., Y.M.

3) Analysis and interpretation of data: M.H., A.N

4) Drafting article: M.H., A.N.

5) Final approval of the version to be submitted: M.H., S.S., Y.M., A.N

\section{Conflicts of interest}

There are no conflicts of interest. 


\section{References}

1. Luke B. The changing pattern of multiple births in the United States: maternal and infant characteristics, 1973 and 1990. Obstetrics and gynecology 1994; 84: 101-6.

2. Rebar RW, DeCherney AH. Assisted reproductive technology in the United States. The New England journal of medicine 2004; 350: 1603-4.

3. Ferraretti AP, Goossens V, de Mouzon J, Bhattacharya S, Castilla J A, Korsak $\mathrm{V}$, et al. Assisted reproductive technology in Europe, 2008: results generated from European registers by ESHRE. Hum Reprod 2012; 27: 2571-84.

4. Practice Committee of American Society for Reproductive Medicine; Practice Committee of Society for Assisted Reproductive Technology. Criteria for number of embryos to transfer: a committee opinion. Fertil Steril 2013; 99: 44-6.

5. Andersen AN, Goossens V, Ferraretti AP, Bhattacharya S, Felberbaum R, de Mouzon J, et al. Assisted reproductive technology in Europe, 2004: results generated from European registers by ESHRE. Hum Reprod 2008; 23: 756-71.

6. Pandian Z, Templeton A, Serour G, Bhattacharya S. Number of embryos for transfer after IVF and ICSI: a Cochrane review. Hum Reprod. 2005;20(10):2681-7.

7. Kjellberg AT, Carlsson P, Bergh C. Randomized single versus double embryo transfer: obstetric and paediatric outcome and a cost-effectiveness analysis. Hum Reprod. 2006;21(1):210-6.

8. Heijnen EM, Eijkemans MJ, De Klerk C, Polinder S, Beckers NG, Klinkert ER, et al. A mild treatment strategy for in-vitro fertilisation: a randomised non-inferiority trial. The Lancet. 2007;369(9563):743-9.

9. [Internet] Ethics Committee of Japan Society for Reproductive Medicine. guidelines in number of the embryos transferred for prevention of multiple pregnancy.

http://www.jsrm.or.jp/guideline-statem/guideline_2007_01.html.

10. Ethics Committee of Japan Society for Reproductive Medicine. guidelines in number of the embryos transferred for prevention of multiple pregnancy. Journal of Japan Society for Reproductive Medicine 2008; 53: 18.

11. [Internet] Japan Society of Obstetrics and Gynecology. Prevention of multiple gestation associated with infertility therapy: The Committee opinion. http://www.jsog.or.jp/ethic/H20_4_tataininshin.html.

12. Matsuda Y, Kawamichi Y, Hayashi K, Shiozaki A, Satoh S, Saito S. Impact of maternal age on the incidence of obstetrical complications in Japan. J Obstet Gynaecol Res 2011; 37: 1409-14.

13. Hayashi M, Nakai A, Satoh S, Matsuda Y. Adverse obstetric and perinatal outcomes of singleton pregnancies may be related to maternal factors associated with infertility rather than the type of assisted reproductive technology procedure used. Fertil Steril 2012; 98: 922-8.

14. Adamson GD, de Mouzon J, Lancaster P, Nygren KG, Sullivan E, Zegers-Hochschild F. World collaborative report on in vitro fertilization, 2000. Fertil Steril 2006; 85: 1586-622.

15. Pinborg A. IVF/ICSI twin pregnancies: risks and prevention. Hum Reprod Update 2005; 11: 575-93.

16. Templeton A, Morris JK. Reducing the risk of multiple births by transfer of two embryos after in vitro fertilization. The New England journal of medicine 1998; 339: 573-7.

17. Jain T, Missmer SA, Hornstein MD. Trends in embryo-transfer practice and in outcomes of the use of assisted reproductive technology in the United States. The New England journal of medicine 2004; 350: 1639-45.

18. Sazonova A, Kallen K, Thurin-Kjellberg A, Wennerholm UB, Bergh C. Neonatal and maternal outcomes comparing women undergoing two in vitro fertilization (IVF) singleton pregnancies and women undergoing one IVF twin pregnancy. Fertil Steril 2013; 99: 731-7.

19. Practice Committee of American Society for Reproductive Medicine. Multiple gestation associated with infertility therapy: an American Society for Reproductive Medicine Practice Committee opinion. Fertil Steril 2012; 97: 825-34

20. Thurin A, Hausken J, Hillensjo T, Jablonowska B, Pinborg A, Strandell A, et al. Elective single-embryo transfer versus double-embryo transfer in in vitro fertilization. The New England journal of medicine 2004; 351: 2392-402.

21. Gerris J, De Neubourg D, Mangelschots K, Van Royen E, Van de Meerssche M, Valkenburg M. Prevention of twin pregnancy after in-vitro fertilization or intracytoplasmic sperm injection based on strict embryo criteria: a prospective randomized clinical trial. Hum Reprod 1999; 14: 2581-7.

22. Gerris J, De Neubourg D, Mangelschots K, Van Royen E, Vercruyssen M, Barudy-Vasquez J, et al. Elective single day 3 embryo transfer halves the twinning rate without decrease in the ongoing pregnancy rate of an IVF/ICSI programme. Hum Reprod 2002; 17: 2626-31.

23. Grady R, Alavi N, Vale R, Khandwala M, McDonald SD. Elective single embryo transfer and perinatal outcomes: a systematic review and meta-analysis. Fertil Steril 2012; 97: 324-31.

24. van Montfoort AP, Fiddelers AA, Land JA, Dirksen CD, Severens JL, Geraedts $\mathrm{JP}$, et al. eSET irrespective of the availability of a good-quality embryo in the first cycle only is not effective in reducing overall twin pregnancy rates. Hum Reprod 2007; 22: 1669-74

25. Veleva Z, Karinen P, Tomas C, Tapanainen JS, Martikainen H. Elective single embryo transfer with cryopreservation improves the outcome and diminishes the costs of IVF/ICSI. Hum Reprod 2009; 24: 1632-9.

26. Roberts SA, McGowan L, Mark Hirst W, Vail A, Rutherford A, Lieberman BA, et al. Reducing the incidence of twins from IVF treatments: predictive modelling from a retrospective cohort. Hum Reprod 2011; 26: 569-75.
27. [Internet] Centers for Disease Control and Prevention. 2009 Assisted Reproductive Technology Success Rates National Summary and Fertility Clinic Reports. http://www.cdc.gov/art/ART2009/

28. [Internet] Japan Society of Obstetrics and Gynecology Ethics Committee. Ethics committee report. http://www.jsog.or.jp/activity/report.html.

29. Toledo MG. Is there increased monozygotic twinning after assisted reproductive technology? Aust N Z J Obstet Gynaecol 2005; 45: 360-4

30. Kawachiya S, Bodri D, Shimada N, Kato K, Takehara Y, Kato O. Blastocyst culture is associated with an elevated incidence of monozygotic twinning after single embryo transfer. Fertil Steril 2011; 95: 2140-2.

31. Moayeri SE, Behr B, Lathi RB, Westphal LM, Milki AA. Risk of monozygotic twinning with blastocyst transfer decreases over time: an 8-year experience. Fertil Steril 2007; 87: 1028-32.

32. Skiadas CC, Missmer SA, Benson CB, Gee RE, Racowsky C. Risk factors associated with pregnancies containing a monochorionic pair following assisted reproductive technologies. Hum Reprod 2008; 23: 1366-71. 